\title{
Performance Analysis of Smart Stick Designed for Visually Impaired People Using GSM and GPS
}

\author{
N. Amutha Priya ${ }^{1}$, S. Sahaya Sathesh Raj ${ }^{2}$, K. Siva ${ }^{3}$, R. Vetha Jebarson ${ }^{4}$ and P. Vignesh ${ }^{5}$ \\ ${ }^{1}$ Assistant Professor, ${ }^{2,3,4 \& 5}$ Student, ${ }^{1,2,3,485}$ Department of Electrical and Electronics Engineering, \\ Rohini College of Engineering and Technology, Tamil Nadu, India \\ E-mail: a.amazing13@gmail.com
}

\begin{abstract}
Visual impairment burdens the people heavily in today's fast-moving culture. Although there are several measures for this impairment, many people skip such chances in fear of their economic condition. In view of helping those people, a sophisticated stick is designed using sensors like ultrasonic sensor, IR sensor and Arduino ICs. Electronically designed walking stick helps to trace the locations using GPS module and to avoid collisions through detection of objects at a certain range of distance from the person in all directions. Sensor placed beneath the bottom of the stick enables the identification of pits/hindrances on the ground. The visually impaired person will be alerted with a voice message generated using the voice recorder which records the response of all the sensors operating in different tracks. In this paper, GSM and sensors together have initiated the role of smart walking stick in the life of several human kinds.
\end{abstract}

Keywords: Visual Impairment, Sensor, GPS, GSM, Smart Stick, Ultrasonic

\section{INTRODUCTION}

Blindness is a sickness which causes hindrance to most of the people's day-to-day activities in this smart moving world. Technological adaptness is not a possible one for all the people specifically, the financially backed and rural ones. Normally, they seek the help of trained dogs or wooden stick as their companion during their travel. This situation has to be changed in order to improve their life style in accordance with the developing technology. Sensors are normally the base for the activation of any control system. This basic tool is completely utilized for detection under a diversified notion like measurement of distance from an object/person, pits/stones on ground, etc [1-5]. These features enhance the utilization of the designed smart stick and completely satisfy the requirements of the visually impaired people to a greater extent. Navigation is easy for visually disabled people with the usage of tool like blind stick, which is indeed a unique and effective one. Normally, visually impaired people are dependent on other human beings or some animals like trained dogs or a wood stick for their movement indoor or outdoor. An innovative blind stick that allows visually challenged people to navigate with ease using advanced technology is proposed in this work. Safety features with GSM Module to help the guardian of the visually aided people to track their location [6-8]. This is designed in a way of receiving an SMS about the location to their phone if the person is lost somewhere else or is in a panic situation. The stick is also efficiently designed to work in rainy environment through total module fabrication. The work is also framed to act as voice-based walking stick which utilizes the programmed voice mainly for guidance. This framework uses GSM technology for navigation purpose [9-10].

\section{SYSTEM ARCHITECTURE}

Fig. 1 indicates the principle of working of the ultrasonic blind stick. This principle is used as a base for designing the proposed blind stick.

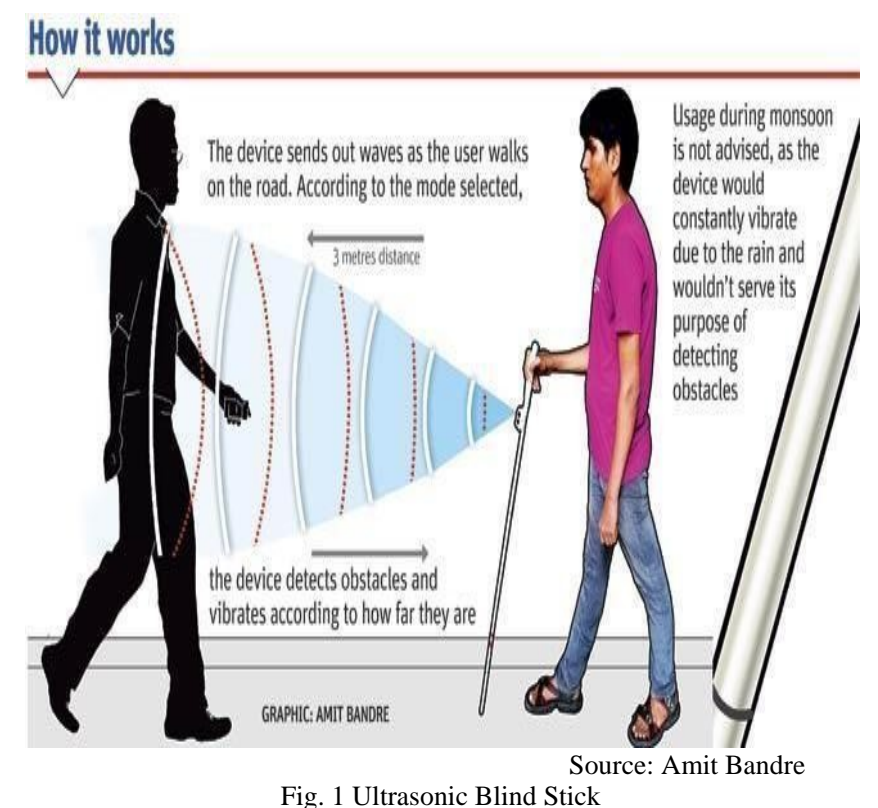

The basic function of ultrasonic sensor is to calculate the distance of an object. In this project, an ultrasonic sensor is used to measure the distance between the nearest obstacles and the blind person. The sensor is able to warn the blind when facing any dangerous situations. The ultrasonic sensor works on the principle of generating high frequency sound waves from the echo pin and evaluates the echo which is received back by the receiver of the sensor. The sensor determines the time interval between sending of the signal and receiving of the echo to calculate the distance of the nearest obstacle. The calculated distance is sent to the Raspberry Pi microcontroller and it speaks the distance of the nearest object to the blind person. 


\section{BLOCK DIAGRAM}

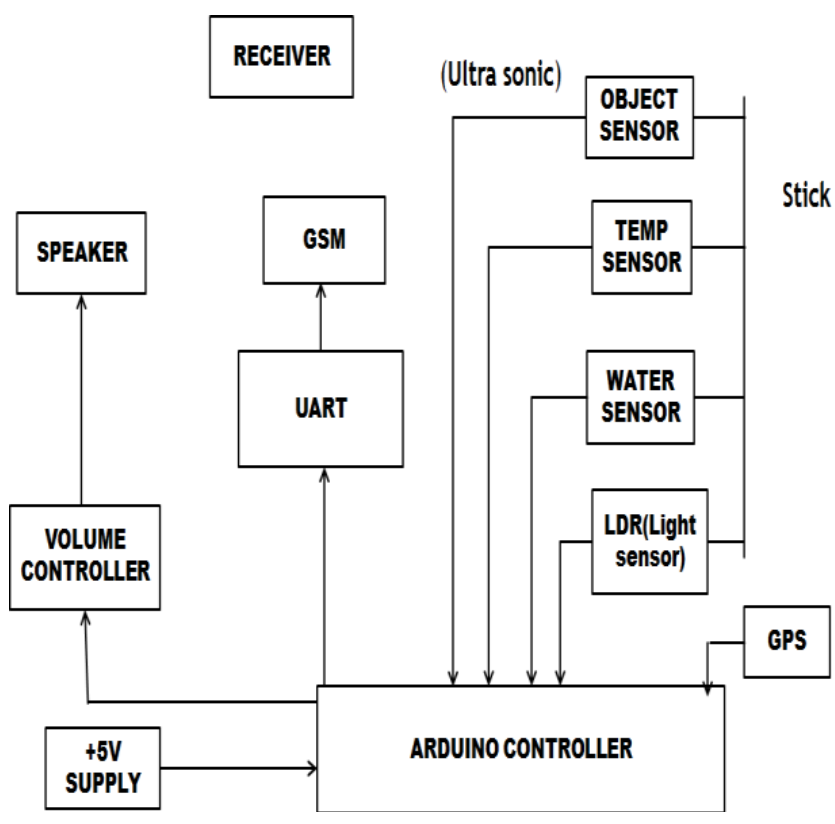

Fig. 2 Block Diagram of Proposed System

The proposed prototype as shown in Fig. 2 is an electronic device that constitutes of obstacle detection module, a fall detection module, hazard detection module, navigation module combined with GPS and IOT, alert system along with distress button and the main processor used here is powered by an external battery. Arduino Uno is the hardware which can execute the instructions that we design in the LabVIEW which is the software which acts as the interface between hardware components and Arduino Uno.

All the mentioned components are combined and mounted on to a walking stick. The project also incorporates IoT technology to interconnect the fall detection, obstacle detection, and hazard detection modules with the internet and thus report any instances of a fall and/or abnormal conditions which the holder may have to face to the family member(s) of the vulnerable person via Email through LabVIEW software. A special distress button will be used by the vulnerable person in a situation where they come face to face with dangers unexpectedly, to connect with the family immediately.

The five main components in the system that is described in the research are GPS module, Ultrasonic Sensor, Accelerometer, Arduino microcontroller and a control unit. Referring to the above fig. 1.2, ultrasonic sensor will send ultrasonic waves, which will bounce back when they will strike with an object or an obstacle in the path of the visually impaired person. The signal which is received back will be sent to the control unit which consists of a microprocessor, Arduino Uno. Arduino will do the calculation and then it triggers the text to speech module as the indicators to warn the blind person of the obstacle. The performance of a walking assistant for the visually impaired depends on some features. The features that we consider here are the types of capturing device used, types of feedback signal provided, the coverage area, the weight, and the cost. These features are key concerns for measuring the efficiency and reliability of any walking assistant that is developed for visually impaired people. The performances of the reviewed walking assistants are drawn in Figure 3.2 by considering the previously described features. From the Figure 3.2, no system offers all features to a reasonable degree. Each framework offers something unique over the others but cannot address every feature because a perfect framework ought to have every one of the features and numerous functionalities. The most significant conclusion is that no framework can fully assist visually impaired people.

The developed systems cannot fulfil user demand perfectly because some of the systems are in their early stage or research stage, and some of them take a long time to perform an experiment with a real-time environment; moreover, some of them are unwearable, heavy, and costly.

Furthermore, the progress and maturity of a navigation assistance system can be measured by how much the system assists the users. Given many hindrances (e.g., front, left, and right obstacles; pothole and hump on roads; and staircases) on the way of walking, most of the systems work in fewer directions. Some of them can detect obstacles in front of the users, some can detect only potholes or humps on the road, and some can detect knee- or head-level obstacles. However, no article thus far has covered all or majority of the hindrances on the way of walking. Hence, we argue that most of the systems are at the early stage of problem solving in relation to this area. The prototypes mentioned in cameras and sensors as capturing devices. Some systems provide tactile feedback, and some provide audio and tactile feedback. The framework developed in works in outdoor scenarios but is unsuitable at indoor.

The system can detect static obstacles within a $5 \mathrm{~m}$ distance but is inappropriate for dynamic obstacles. Some systems provide indoor coverage, whereas some provide outdoor coverage. Nevertheless, the user demand is indoor and outdoor. In accuracy comparison, the highest and lowest accuracies achieved by the system were approximately $98.8 \%$ when the obstacle was close $(5 \mathrm{~cm})$ to users and $62.8 \%$ when the obstacle was far $(350 \mathrm{~cm})$ away from the users. The highest and average accuracies obtained by the system proposed were approximately $98 \%$ and $96 \%$, respectively.

Most of the pixel-wise accuracy within different ranges was over $90 \%$. Avoidance algorithm without ultrasonic sensor had an accuracy of $98.93 \%$ in case of frosted glass but had extremely low accuracy in case of pure transparent glass. The detection rate obtained was $94.4 \%$. Hence, the developed walking assistants have achieved sufficient accuracy when obstacles are close to users. 


\section{TOOLS USED IN AIDING VISUALLY IMPAIRED PEOPLE}

Smart stick that is low cost but durable and assists visually impaired people in their movements. The stick provides pure knowledge about the distance and the location of the obstacle through vibration and audio in hand and ear, respectively. Bluetooth dongle has been used to establish a connection between the earphone and the smart stick. The proposed scheme can detect static and dynamic obstacles, downstairs, and upstairs. The main hardware components used in the system are a microcontroller, an ultrasonic sensor, and HC-05 master-slave Bluetooth modules. However, the smart stick cannot detect objects in all surroundings, that is, it can only detect obstacles in front of users, and the error rate becomes high with the increment in distance. Walking assistant called AKSHI to aid visually impaired people. The system uses Raspberry Pi 2, ultrasonic sensor, GSM module, and RFID reader and tags. The RFID reader is attached with the bottom part of the stick that can detect the obstacles using RFID tags. The ultrasonic sensor is placed below the circuit box on the track of $45^{\circ}$ angles.

Another box, including Raspberry Pi, GSM, and GPS modules, is attached to the stick. A mobile application was also developed to keep track of the user location using GSM and GPS modules. Users can access the GPS location interface that displays the current location of the visually impaired. However, the RFID tags work within a short range, and the stick cannot work in dirty and muddy environments. Electronic mobility cane for way finding and obstacle detection of visually impaired people. The system constructs a logical map of the surroundings and maintains them based on priority. The system provides the priority information to users by using feedback signals, such as voice, vibration, or audio, and detects staircase and floor statuses. The system consists of ultrasonic sensors, liquid, metal detection sensor, wireless transceivers, battery, and microcontroller circuits. Furthermore, the system collects and categorizes the information of surroundings.

However, the system cannot identify overhanging obstacles and requires excessive training that might be expensive for visually impaired people. Electronic tool integrated with a conventional cane that provides an alarm signal when obstacles are detected. A custom-built printed circuit board embedded with a microcontroller drives the sensor and the motor. Its weight with battery is approximately $110 \mathrm{~g}$.

The system calculates the distance during the notification of obstacles. An alarm signal is generated when the calculated distance is between $0.2 \mathrm{~m}$ and $0.6 \mathrm{~m}$, and another signal is generated when the distance is between $0.6 \mathrm{~m}$ and $1 \mathrm{~m}$. Moreover, the system searches for another obstacle when no obstacles are observed between $0.2 \mathrm{~m}$ and $1 \mathrm{~m}$. Smart Eye to aid visually impaired people by providing them with information on the surroundings. The system has two modules, namely, embedded wearable sensor and Smartphone modules. The wearable sensor module consists of power (9 V DC battery), CPU (32 b m bed NXP LPC1768), sensors (ultrasonic and motion sensors), and communication (Bluetooth or Wi-Fi chip) parts. An Android application was developed to provide distance notification to users that is returned by using an ultrasonic sensor. The embedded module and Smartphone communicate through Bluetooth or Wi-Fi. The developed application is vigorous and detects obstacles at approximately $10 \mathrm{ft}$ away. This process is conducted in a laboratory and cannot interact with the real world along with real-time obstacles. Sonic Eye based on ultrasonic echolocation and spatial hearing principles that aid visually impaired people for their safe mobility. The tool comprises an ultrasonic emitter, stereo microphones, and a wearable headset. The resonance of ultrasonic pulses is noted, and a feedback signal (human auditory range) is then provided to the users with the presence of obstacles.

The tool can be used efficiently to observe the surroundings, and the human auditory structure may swiftly adapt to artificial echolocation signals. However, the system is not precisely contactless as the users should wear the echolocation device. Visually impaired people to aid them in walking by identifying an object and creating an audio signal as per the distance of the object. The system is designed similar to a spectacle and can detect obstacles under $3 \mathrm{~m}$ distance and $60^{\circ}$ angle. The spectacle consists of an ultrasonic sensor, a microcontroller, and an alarm generator.

The ultrasonic sensor generates an ultrasound wave that is reflected to the sensor by observing the obstacle's location within $3 \mathrm{~m}$. The microcontroller measures the distance by manipulating the time between the back and forth travel of ultrasound from the obstacle. A signal is transferred to the alarm generator with the presence of obstacles, and it generates an alarm based on the signal from the microcontroller. The ultrasonic sensor is positioned at the middle of the spectacle, and the microcontroller, alarm generator, and battery are attached to the temple. The developed scheme is a low-cost and straightforward strategy but bulky. Temperature, density, and weight also limit the ultrasonic sensor.

Moreover, system accuracy varies at near and far distances. Visually impaired people that can be used as an artificial eye. The embedded tool in the proposed system is used to detect obstacles using ultrasonic sensors, and vibrator motors generate the feedback. An Android device is used for navigation. An Android application is used to track the position of blind people using GPS and General Packet Radio Services (GPRS) and guide them in the proper direction. The system is used to detect and locate obstacles.

\section{HARDWARE DESCRIPTION}

The apparatus which we have picked is Arduino uno, which have three ports in particular A, B and C which can send and get signals from sensors and hardware required. Forty 
advanced I/O lines generally speaking with help for different conventions like SPI, PWM out, quadrature encoder input, UART(Tx and Rx), and I2C for simpler correspondence with sensors; eight single-finished simple information sources; two differential simple data sources; four single-finished simple yields; and two groundreferenced simple yields take into consideration availability to endless sensors and gadgets and automatic control of frameworks. The majority of this usefulness is implicit and preconfigured in the default FPGA usefulness, which kills the requirement for extension sheets or "shields" to include utility. At last, these highlights enable understudies to do true designing right now from radio controlling vehicles to making remain solitary restorative gadgets. So, this brilliant stick would likewise progress toward becoming independent and free. The sensor modules that have been selected out are. The sensor modules that have been selected out are:

1. Ultrasound Sensor.

2. Water Sensor.

3. 3-axis accelerometer inbuilt in Arduino Uno.

A portion of alternate prerequisites are GPS module, trouble catch we can use the catch which is available on Arduino Uno for this capacity, Buzzer, additionally alerts can likewise be given out utilizing the sound out port which is available on Arduino Uno subsequent to programming it as indicated by the condition required. The GPS module used is based on the Ublox NEO6M. This unit uses the latest technology from Ublox to give the best possible positioning information and includes a larger built-in 25 x $25 \mathrm{~mm}$ active GPS antenna with a UART TTL socket.

The power supply to this device is through a $12 \mathrm{~V}$ battery with $9000 \mathrm{mAh}$ which is connected externally. The most crucial part of this project is to interface different types of sensors with Arduino Uno and to monitor the communication of different types of data like analog and digital between Arduino Uno and sensors.

It has $\mathrm{I} / \mathrm{O}$ pins consisting of serial port protocols such as SPI, I2C, and UART (Tx and Rx), which make it easier to communicate with the sensor and peripheral modules. Also, the data from the I/O pins can be accessed directly without the need of writing any code and can be displayed or processed directly. Since, Arduino Uno can be connected through $\mathrm{Wi}-\mathrm{Fi}$, this can be used to send the warnings to the relatives of the user through the mail using SMT protocol in case of emergencies. So, there is no need to use another GSM module for providing connectivity to the user with an IoT server.

\section{SOFTWARE DESCRIPTION}

Lab VIEW is a graphical programming condition that can be utilized to rapidly create applications that scale over different stages and OSs. The intensity of Lab VIEW is in its capacity to interface with a great many gadgets and instruments utilizing several inherent libraries and prebuilt
VIs to enable you to quicken improvement time and rapidly secure, examine, and present information. Instead of investing bountiful measures of energy investigating code sentence structure or creating UIs, understudies can utilize the Lab VIEW software to focus on constructing their systems and solving their design problems without the added pressure of a burdensome tool. Applications in Lab VIEW mimic the appearance of real instruments (like multimeters, signal generators, or oscilloscopes), so they are called virtual instruments or VI_s Every Lab VIEW application has a front panel, an icon/connector pane, and a block diagram. The front panel serves as the imitation of the real-world user interface of the device that the VI is defining. In summary, Lab VIEWV Is are graphical, driven by dataflow and event-based programming, and are multitarget and multiplatform capable.

They also have object-oriented flexibility and multithreading and parallelism features. Lab VIEWVIs can be deployed to real-time and FPGA targets. So, we design the required VI in Lab VIEW and connect it to RIO for the execution of the connected hardware component, thus obtaining the required prototype.

\section{METHODOLOGY}

\section{A. Obstacle Detection}

1. Both the ultrasonic sensors are in standby mode.

2. Sensors are in active mode.

3. If any immovable object is present within the range of half meter, then the ultrasonic sensor detects it and sends this to the Arduino Uno, which then instructs the buzzer to warn the user.

4. If any living being is found in the range of three to seven meters (adjustable) and in 120 degrees angle then it detects their heat signatures and transmits the same information to Arduino Uno, which instructs the buzzer to warn the user.

\section{B. Fall Detection}

1. The inbuilt accelerometer starts its operation.

2. If the orientation of the accelerometer changes more than the threshold value, then it assumed to a result of a fall.

3. The selected list of contacts of the user are alerted regarding the fall through the mail.

\section{RESULTS AND DISCUSSION}

The proposed design of smart stick is displayed as shown in Fig. 3. The working of alert system in the smart stick is indicated in Fig. 4. The GPS location tracker's impact is shown in Fig. 5. This alert-based system sends a notification to the neighbours' mobile phone which is connected to the stick. The notification shows that there is an emergency alert for the user. The above figure shows how the notification is received by the user. 

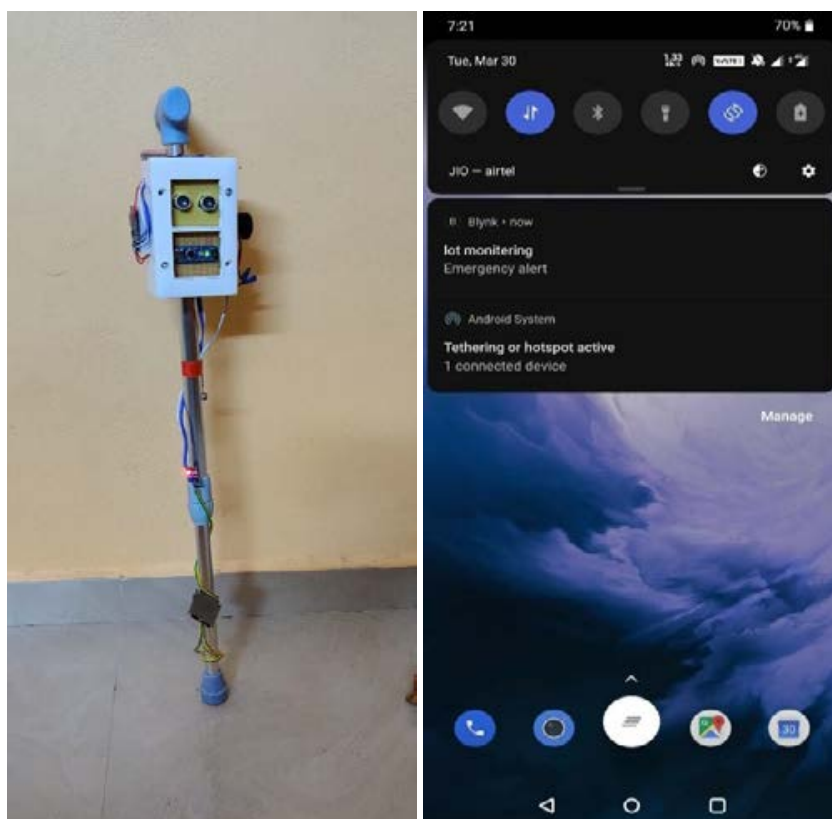

Fig. 3 Smart Stick
Fig. 4 Alert System

The second important feature of our electronic stick is that it shows the live location of the blind stick. This is done by the GPS tracking device fixed on the stick. By using this feature the location of the blind person can be easily tracked in times of emergency and they can be found easily. This has a buzzer system in which a buzzer sound comes while facing obstacles. The pattern of buzzer sound differs with the distance between the stick and the obstacle. This also has a water sensor which gives a buzzer when there is water in the way of the blind people.

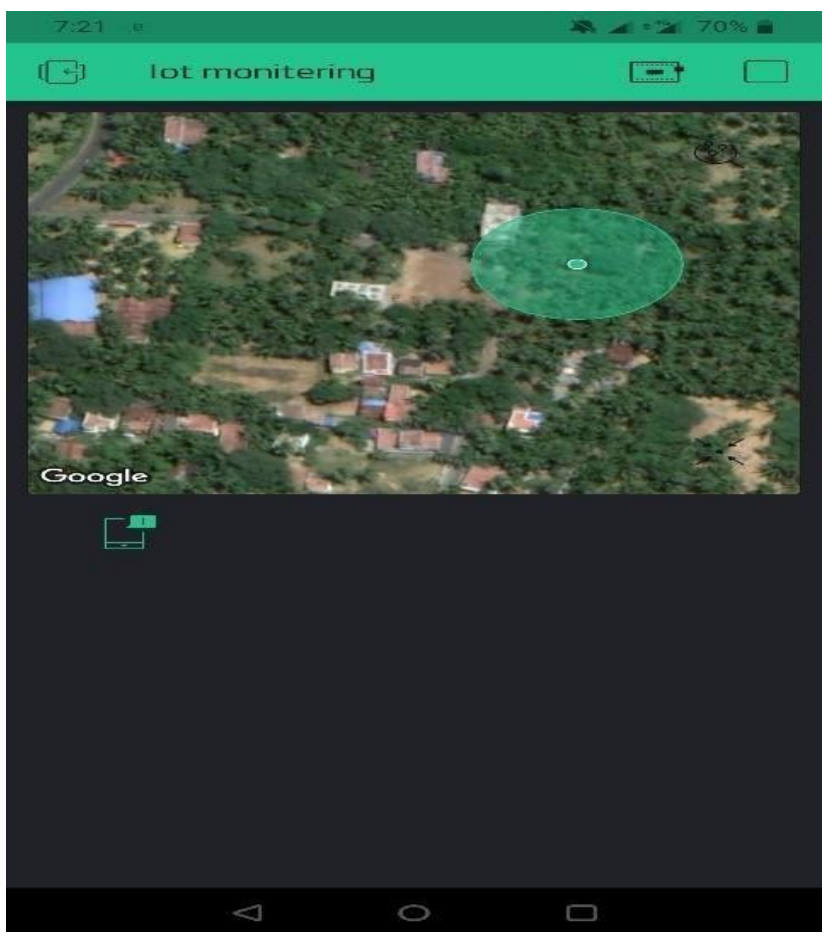

Fig. 5 GPS Locator

\section{CONCLUSION}

A prototype of an electronic system that helps an assailable/unsafe person to be connected with alert his/her family in times of danger is designed in this work. While most appliances marketed as smart walking stick incorporate only GPS connectivity with some basic features, our project envelopes fall detection, hazard detection, obstacle detection and combine these with the Internet of Things to give a practical solution that puts the technology in service to those in need. In the future, this project can be extended by interfacing a camera to the walking stick. The live video feed of the camera is processed continuously using various digital image processing techniques and by connecting it to a cloud, the surroundings of the person can be estimated and instructions can be given according to it. Whereas this requires High Definition camera, continuous connectivity to high-speed internet, complex data processing techniques, high-speed processor and a cloud server in a remote location.

\section{REFERENCES}

[1] Aboubakr Aqle, Kamran Khowaja and Dena Thani, "Preliminary evaluation of Iterative Search Engine Interface for Visually Impaired Users,” IEEE access, Vol. 8, pp. 45061-45070, 2020.

[2] Bin Jiang and Jianchen Yang, "Wearable Vision Assistance System Based on Binocular Sensors for Visually Impaired Users", IEEE Internet of Things Journal, Vol. 6, No. 2, pp. 1375-1383, 2019.

[3] Chanhumpark, Se-Wooncho, JihoChoi and Kang-young, "Deep Feature Based Three Stage Detection of Banknotes and Coins for Assisting Visually impaired people,” IEEE access, Vol. 8, pp. 18458184613, 2020.

[4] Dannile Croce, Laura Giarre and Ilenia Tinnirela, "An Indoor and Outdoor Navigation System for Visually Impaired People," IEEE access, Vol. 8, pp. 170406-170418.

[5] Guojun Yang and Jafar Saniie, "Sight-to-sound Human Machine Interface for Guiding Visually Impaired People,” IEEE access, Vol. 8, pp. 185416-185428, 2020.

[6] Jean Connier, Haiying Zhou and Phillippe Vaslin, "Perception Assistance For The Visually Impaired Through Smart Objects,” IEEE acess, Vol. 8, pp. 46931- 46945, 2020.

[7] Md Milon and Muhammad Sheik Sadi, "Developing Walking Assistants for Visually Impaired People”, Vol. 19, No. 8, pp. 28142828, 2019.

[8] Shabnam Mohamed Aslam and Shirina Sameera, "Gesture Recognition Algorithm for Visually Impaired People Using Crow Search Method,” IEEE access, Vol. 8, pp. 63144-63161, 2020.

[9] Vidula Meshram and Kailas Patil, "An Astute Assistive Device for Mobility and Object Recognition for Visually Impaired people,” IEEE Transaction on Human Machine systems, Vol. 49, No. 5, pp. 449-460, 2019.

[10] Wan-Jung Chang, Linag-Bi Chen and Ming-Chechen, "Design and Implementation of an intelligent Assistive System for Visually Impaired for Aerial Obstacle Avoidance and Fall Detection,” IEEE Sensors Journal, Vol. 20, No. 17, pp. 10199-10210, 2020. 